\title{
Post high intensity pull-over semi-tethered swimming potentiation in national competitive swimmers
}

\author{
Francisco CUENCA-FERNÁNDEZ 1 *, Nuno M. BATALHA 2, 3, Jesús J. RUIZ-NAVARRO 1, \\ Esther MORALES-ORTIZ 1, Gracia LÓPEZ-CONTRERAS 1, Raúl ARELLANO ${ }^{1}$
}

${ }^{1}$ Aquatics Lab, Department of Physical Education and Sports, Faculty of Sport Sciences, University of Granada, Granada, Spain; 2Department of Sports and Health, University of Evora, Evora, Portugal; ${ }^{3}$ CHRC-UE - Comprehensive Health Research Care, University of Evora, Evora, Portugal

*Corresponding author: Francisco Cuenca-Fernández, Aquatics Lab, Department of Physical Education and Sports, Faculty of Sport Sciences, University of Granada, Carretera de Alfacar, 18011 Granada, Spain. E-mail: cuenca@ugr.es

\begin{abstract}
A B S T R A C T
BACKGROUND: The swimming community has shown considerable interest in using dry-land warm-ups as a method of impacting performance. This study compared the effects of high-resistance pull-over and swimming warm-up in semi-tethered resisted swimming.

METHODS: An incremental-load semi-tethered swimming test was individually administered in 20 national-competitive swimmers to determine the load maximizing swimming power. In different sessions, participants tested such a load 6 min after a swimming warm-up (SWU) or a dry-land warm-up (DLWU: 3 pull-over reps at $85 \%$ of the one-repetition maximum). Kinetic variables (velocity, force, acceleration, impulse, power rate of force development [RFD] and intra-cycle variation), were obtained with a linear encoder through trapezoidal integration regarding time. Kinematic variables (distance, time, stroke-rate and stroke-length), were obtained by video recordings. The differences between protocols were observed by paired-samples $t$-test (ANOVA). Pearson's coefficient explored correlations between kinetics and kinematics variables; significance was set at $\mathrm{P}<0.05$.

RESULTS: DLWU increased RFD $(34.52 \pm 16.55 v s .31 .29 \pm 13.70 \mathrm{~N} / \mathrm{s} ; \Delta=9.35 \%)$ and stroke-rate $(64.70 \pm 9.84 v s .61 .56 \pm 7.07 \mathrm{~Hz} ; \Delta=5.10 \%)$ compared to SWU, but decreased velocity, force, acceleration, impulse and power. During the incremental-load test velocity and power were higher than obtained after SWU $(1.21 \pm 0.14 v s .1 .17 \pm 0.12 \mathrm{~m} / \mathrm{s} ; \Delta=3.06 \%),(51.38 \pm 14.93 v s .49 .98 \pm 15.40 \mathrm{~W} ; \Delta=2.72 \%)$, suggesting enhancements prompted by the test itself. Correlations between stroke-length with impulse $(r=0.76)$ and power $(r=0.75)$ associated kinetics with kinematics.

CONCLUSIONS: Potentiation responses were present after the dry-land warm-up. However, swimmers may benefit more from submaximal prolonged conditioning activities such as resisted swimming rather than high-resistance dry-land sets to obtain performance enhancements.

(Cite this article as: Cuenca-Fernández F, Batalha NM, Ruiz-Navarro JJ, Morales-Ortiz E, López-Contreras G, Arellano R. Post high intensity pullover semi-tethered swimming potentiation in national competitive swimmers. J Sports Med Phys Fitness 2020;60:1526-35. DOI: 10.23736/S00224707.20.11136-8)

KEY WORDS: Sports; Exercise; Muscle fatigue; Physical exertion.
\end{abstract}

$\mathrm{T}$ he use of high-intensity conditioning exercises (CEs), as a method of short-term enhancements in the subsequent task, has been reported in the current literature as post-activation performance enhancement (PAPE). ${ }^{1}$ After recent contractile history, muscles are in both a potentiated and fatigued state. However, fatigue dissipates faster than potentiation, creating an augmented muscular prevalence for possible performance enhancement. ${ }^{2}$ Several mechanisms may influence PAPE, including the effect of elevat- ing the muscle temperature on the cross-bridge cycling rates; $;, 4$ the increases in motoneuron excitability detected after voluntary contractions; 5,6 or the increase in circulating hormones as epinephrine or norepinephrine after brief bouts of intense exercise. ${ }^{7}$

The training PAPE principles are based on complex training, ${ }^{8}$ which consists of providing a resistive $\mathrm{CE}$ as similar as possible to the real action before performing a sport-specific activity involving the same muscle groups 


\section{COPYRIGHT $^{\circledR} 2020$ EDIZIONI MINERVA MEDICA}

at a later time. ${ }^{5}$ Considering that some dry-land exercises involving mainly pulling actions have been shown to be effective predictors of swimming performance, ${ }^{9-12}$ this led some authors to test them as means to create CEs to potentiate swimming. However, the results were marginally unsuccessful. ${ }^{13}, 14$ The swimmer's performance does not depend solely on the capacity of the muscle system to produce large amount of power, but also on the ability to transfer it into the water to create effective propulsion. ${ }^{15-17}$ Therefore, it is still uncertain if swimmers may benefit from these methods to increase performance.

One critical aspect of using pulling-CEs is that the underwater path in swimming is not in a straight line from the front to the back. ${ }^{17}$ As swimmers search for steady water using a certain amount of sculling like movements to create effective propulsion, the capacity of a dry-land pulling-CE to reproduce the specific movements of the underwater arm-pull could be questioned. ${ }^{15,} 18$ Nevertheless, a fluid dynamics study reported that contrary to accepted notions in swimming, pronounced lateral movements could decrease the contribution of drag forces to thrust, reducing the effectiveness of the arm-pull. ${ }^{19}$ Therefore, this could provide an argument in favor of keep trying this kind of procedures with swimmers.

The analysis of the literature reveal that some other resistance training approaches have been shown to have a positive impact on swimmers, even without applying biomechanically-similar CEs. ${ }^{15,20,21}$ In addition, the main regulatory PAPE responses have been recently unrelated to the localized effects caused by post-activation potentiation (PAP), 5,22 a muscle response mechanism originated by the contraction-induced effects in the muscle-myosin head phosphorylation. ${ }^{23}$ Thus, there is no evidence supporting the need to achieve full simulation of the real movement during conditioning protocols to induce PAPE, but rather evidence in favor of sufficient stimulation of the muscle system to achieve those responses. , $^{1,5,24}$

On the other hand, the common limitations found in some PAPE-swimming studies are that the conclusive assumptions are based solely on the effects of the CEs on the kinematic variables of swimming (velocity, distance, time, stroke-rate and stroke-length), $13,14,25,26$ while the kinetic variables (force, acceleration, impulse, power and RFD) are rarely evaluated or only collected in tethered conditions (i.e. without displacement), ${ }^{27}$ which it limits the possibility of exploring the hypothetical performance enhancements caused by the PAPE effects. Thus, this forces a path in which the biological or physiological effects prompted by the CEs could be biased by an inadequate procedure to detect those changes.
Recently, two potentiation approaches carried out in competitive male swimmers have obtained significant results in kinetic variables measured in real swimming conditions: 1) swimming arm-pull thrust $(\Delta=18.73 \%)$, after an elastic band arm-pull protocol; 16 and 2) in flutter kick thrust $(\Delta=15 \%)$ after an unloaded countermovement jump protocol. ${ }^{28}$ In both studies human thrust was measured through differential pressure sensors placed on hand and/or legs and the improvements in kinetic variables corresponded to low to moderate positive influences on speed $(\Delta=2-10 \%)$, respectively. Consequently, the authors concluded that PAPE protocols could improve swimming performance. While we believe that these proposals have merit and could re-orientate the way to assess potentiation responses in the water, acquiring such sophisticated equipment could be difficult for some population.

Therefore, considering that it would be preferable to test kinetic variables through familiar procedures, ${ }^{16}$ the fact that inspired the assessment tool applied in this study was based on another study that also obtained improvements through a different PAPE approach that could be equally valid for increasing swimmers performance. ${ }^{26}$ The conditioning protocol consisted of $4 \times 10-\mathrm{m}$ maximal semi-tethered resisted swimming efforts and the participants obtained improvements in 100-m freestyle performance $(-0.54 \mathrm{~s})$. In this regard, although the semi-tethered resisted efforts were designed as the $\mathrm{CE}$, it could provide an alternative way of evaluating PAPE responses given its specificity and sensitivity in monitoring the similar muscular activity to that of free swimming. $9,12,26$

For those reasons, the aim of this study was to investigate whether muscular performance could be improved after a dry-land or an aquatic warm-up in semi-tethered resisted swimming (STRS). Our hypothesis was that, if the kinetic energy is transferred directly to the water to produce the swimmer's displacement, then the data collected by STRS would reflect the effective swimmer's propulsion. Consequently, this could shed more light about the presence or not of potentiation responses in the kinetic variables of swimming.

\section{Materials and methods}

\section{Participants}

Twenty competitive male swimmers were fully informed about the experimental procedures and voluntarily provided signed informed consent to participate in this study (mean \pm standard deviation [SD]): $18.02 \pm 1.39$ years; $70.36 \pm 8.97 \mathrm{~kg} ; 1.80 \pm 0.04 \mathrm{~m} ; 74.29 \pm 7.89 \%$ performance 


\section{COPYRIGHT $^{\odot} 2020$ EDIZIONI MINERVA MEDICA}

level of the world record (50-m Freestyle, Short course); FINA points: $477 \pm 163$ points. Swimmers under 18 years of age were asked to provide signed parental consent. The exclusion criteria included were: 1) swimmers without at least one national-level competitive participation in the last year; 2) participants who have suffered any injury or disease in the past 6 months; and 3) no semi-tethered or in-water resisted practice during the last 3 months. To improve the reliability of the measurements, every test was individually assigned at the same time of the day and the experiment was conducted during the second macrocycle of the season. ${ }^{29}$ All participants were asked to refrain from intense exercise or any stimulant drink during the day before. All the procedures were performed in accordance with the Declaration of Helsinki with respect to human research, and the study was approved by the Institutional Review Board of the University (reference: 852).

\section{Experimental design}

A $t$-test design was used to compare the differences of 2 conditioning protocols in a STRS effort. One protocol consisted of a standard swimming warm-up (SWU), which acted as control, composed of 400-m varied swim paces, including $2 \times 50$-m front crawl swim $\left(12^{\prime} 5\right.$ fast $/ 12$ ' 5 smooth); The other protocol consisted in SWU followed by a dry-land warm-up (DLWU), composed of dynamic limb stretching followed by 3 pull-over reps at $85 \%$ of the onerepetition maximum (RM) load. The STRS efforts were conducted 6 min after the experimental warm-ups with the load that maximized swimming power. This load was obtained through an aquatic incremental-load test following the same procedures reported in previous studies. ${ }^{12,30}$ Additionally, the effort producing the maximal swimming power, was extracted to compound a new category which gathered the result achieved after several repetitions of resisted swimming (denoted INCTEST). This category was used to be compared to SWU.

\section{Procedure}

First, all participants visited the laboratory on 2 separate days to randomly conduct one incremental-load strength test in dry-land or in aquatic conditions. Both the in-water and the strength tests were performed on different days $(72 \mathrm{~h})$ to ensure that one test would not affect the other. ${ }^{29}$ The dry-land strength test was carried out to obtain the swimmer's individualized load in the pull-over CE (i.e. DLWU). Its design was based on the fundamentals of strength testing taken from the American College of Sports Medicine guidelines ${ }^{31}$ and was adapted for study purposes

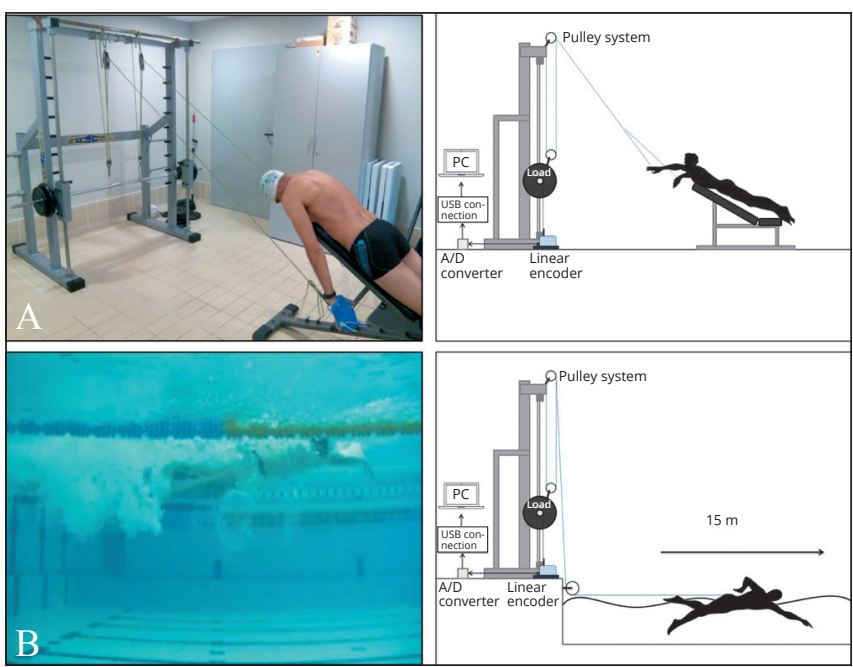

Figure 1.-A) Layout of the dry land; and B) aquatic protocols, designed to evaluate performance of the swimmers through the adaptation of a Smith machine with a linear encoder.

based on previous research. ${ }^{13}$ Participants started the test in prone position on an inclined bench $\left(45^{\circ}\right.$ from vertical), with both arms horizontally extended in front of the body, and each hand holding a handle from a pulley system installed on a Smith Machine (Jim Sports Technology S.L., Lugo, Spain) (Figure 1A). They were asked to perform a complete shoulder extension at maximal velocity, then return to the starting position in a controlled manner, remain in the starting position for $1 \mathrm{~s}$, and perform a second repetition. Every participant had to complete 2 reps with each load (increases of $5 \mathrm{~kg}$ ) every $2 \mathrm{~min}$. The test ended when swimmers were unable to perform a complete repetition, considering this load as the pull-over RM $(38.21 \pm 4.58 \mathrm{~kg})$.

The aquatic incremental-load strength test was individually administered with the purpose to obtain the load that maximized swimming power. According to some authors, $, 9,32$ swimming power expresses reliable information about swimming performance because this variable brings together the force- and velocity-related variables. The test consisted of several front crawl STRS efforts of 15-m and it was conducted in a $25-\mathrm{m}$ indoor pool (water and air temperatures of 28.2 and $28.9^{\circ} \mathrm{C}$, respectively). After performing the SWU, participants started the first effort connected to a Smith machine by a taut rope and a waist belt through a pulley system. As every swimming effort produced the lift of the load, it was possible to gather the variables delivered to that load through the linear encoder (Figure 1B). This protocol was replicated. ${ }^{12}$ The swimmer adopted a frontal extended position next to the edge of 


\section{COPYRIGHT $^{\circledR} 2020$ EDIZIONI MINERVA MEDICA}

the pool, with legs outstretched until the cable was fully extended, without raising the previously set load. On the tester's command, the swimming exercise started at maximum speed up to $15-\mathrm{m}$. Neither pushing-off from the wall, nor breathing, was allowed. All the efforts had time duration between 10 to $20 \mathrm{~s}$. The test started with $1 \mathrm{~kg}$ of load (after the pulley system), and it was increased by successive $1 \mathrm{~kg}$ increments to individually obtain the execution data corresponding to the maximal swimming power load. In order to attempt total recovery, 6 min of rest were given between efforts. ${ }^{12,} 26$

Upon return for a third session, participants were randomly assigned into 2 groups. The first group underwent SWU, while the second group performed DLWU and the effects were tested after 6 min of rest in a front crawl STRS effort with the load that maximized swimming power. Finally, on a fourth day, the group order was reversed to avoid the "fatigue/learning" effect and tests were repeated.

All targeted loads, both in the aquatic and dry-land tests, were adapted and previously tested with an electronic dynamometer (WeiHeng ${ }^{\circledR}$, Guangzhou WeiHeng Electronics Co., Ltd.). To gather the data from every STRS effort to the software application, an isoinertial dynamometer (T-Force Dynamic Measurement System, Ergotech, Murcia, Spain) was used to acquire, display, and process velocity-time data obtained from the lift of the bar of the adapted Smith machine. ${ }^{12}$ All the aquatic registers were synchronized and visually inspected with video recordings taken from 3 cameras installed on 3 underwater portholes along the pool (Sony Video Camera, 50Hz; Sony Electronics Inc., Tokyo, Japan). One of them recorded the underwater phase to 7.5 $\mathrm{m}$, the second recorded from 7.5 to $12.5 \mathrm{~m}$ and the third from 12.5 to $17.5 \mathrm{~m}$. The three sequences were overlapped in space and time by a video switcher (Digital Video Switcher SE-900, Datavideo Technologies Co., Taipei, Taiwan). The first 2 arm-strokes were excluded, and the next 10 consecutive arm-strokes were selected for further analysis. The ICC between measurements was performed by two experts (intra- and inter-measurements). Ten STS efforts were digitized by 2 independent researchers with experience in processing the custom-designed routine. The intra-observer ICC ranged between 0.97 (95\% CI: 0.960.98 ) and 0.98 (95\% CI: 0.97-0.99), and the inter-observer ICC ranged from 0.96 (95\% CI: 0.94-0.98) to 0.97 (95\% CI: 0.95-0.99), for the stroke rate.

\section{Variables measured}

Instantaneous velocity (V) and acceleration (Accel) were acquired from the encoder at a sampling rate of $1000 \mathrm{~Hz}$.
The force delivered to the load (F) was calculated according to Newton's second law $\left(\mathrm{F}=\mathrm{m}^{*} \mathrm{a}\right)$ where $m$ stands for the load lifted on the Smith Machine and $\alpha$ stands for the Accel signal. Data was smoothed using a fourth order Butterworth low-pass digital filter, with a cut off frequency of $10 \mathrm{~Hz}$, defined according to residual error analysis versus cut-off frequency. Through the synchronization of the video recording with the encoder registering, it was possible to detect the slopes produced by every arm-stroke on the Accel signal. Therefore, every slope with values above zero was considered as a one-arm stroke (Figure 2), and the values of the variables were calculated as the means obtained on 10 arm-strokes.

A trapezoidal integration regarding time was used to calculate the absolute values of impulse for each armstroke with a frequency of data acquisition: $1000 \mathrm{~Hz}$. The impulse normalized to the load pulled $\left(\operatorname{Imp}_{\mathrm{REL}}\right)$ was obtained by dividing the absolute values of impulse by the mass of the load pulled $(\mathrm{kg})$ and then this value was averaged to 10 arm-strokes. ${ }^{12}$ The swimming power delivered to the load was calculated as the force multiplied by the velocity delivered and the RFD was calculated as the slope of the force-time curve (RFD $=\Delta$ Force $/ \Delta$ Time) ${ }^{33}$

The stroke-rate (SR) was determined using a frequency measuring function for each 3 -stroke cycle, divided by the time elapsed during this action $(\mathrm{Hz})$, and multiplied by 60 (to obtain the rate in cycle/min). The stroke-length (SL) was determined by dividing the mean $\mathrm{V}$ by the mean SR $(\mathrm{Hz})$. The distance covered (DC) in 10 arm-strokes was provided directly by the encoder and the time to complete

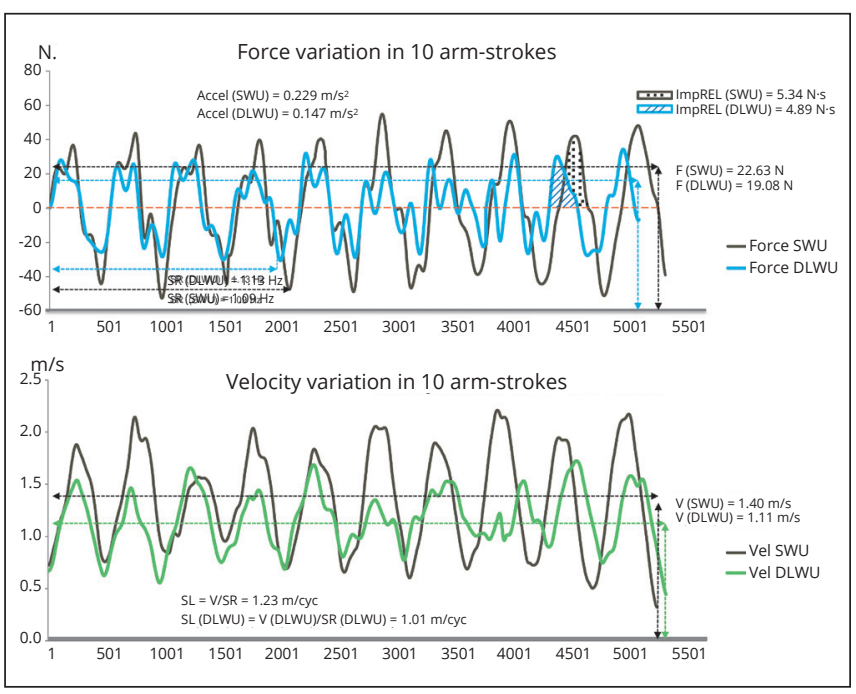

Figure 2.-One case example that demonstrates how PAPE may affect performance of the same swimmer in DLWU compared to SWU. 


\section{COPYRIGHT $^{\odot} 2020$ EDIZIONI MINERVA MEDICA}

5-m (T5m) was calculated as the distance of 5-m divided by the mean $\mathrm{V}$.

The Intra-cyclic Velocity Variation (IVV) was analyzed as described elsewhere. ${ }^{34}$ Where $x$ represents the mean V; $\mathrm{x}_{i}$ represents the instantaneous $\mathrm{V} ; F i$ represents the acquisition frequency $(1000 \mathrm{~Hz})$, and $n$ is the number of measured strokes:

$$
I V V=\frac{\frac{\sqrt{\sum i\left(x_{i}-\bar{x}\right)^{2} \cdot F_{i}}}{n}}{\frac{\sum i x_{i} \cdot F_{i}}{n}} \cdot 100
$$

\section{Statistical analysis}

Descriptive statistics were obtained and expressed as mean $\pm \mathrm{SD}, 95 \%$ confident intervals, relative changes $(\% \Delta)$ and respective effect sizes (d). The effect sizes were categorized as follows: small if $0 \leq|\mathrm{d}| \leq 0.5$, medium if $0.5<|\mathrm{d}| \leq 0.8$, and large if $|\mathrm{d}|>0.8 .{ }^{35}$ After Saphiro-Wilk testing for normality distribution, statistical differences between SWU and DLWU were determined using pairedsamples $t$-test (ANOVA). To detect differences between the protocols, statistical significance was set at the alpha level $\mathrm{P} \leq 0.05$. The same analysis was applied to compare results from INCTEST with results from SWU protocol.
Pearson's correlation coefficient was used to determine the relation between kinetics and kinematics variables in the 3 protocols. All statistical procedures were performed using SPSS 21.0 (IBM Chicago, IL, USA).

\section{Results}

Mean, SD, 95\% confident intervals, mean differences, relative changes $(\% \Delta), \mathrm{P}$ values and effect sizes for all tested semi-tethered swimming variables are presented in Table I. P values and effect sizes from $t$-test (ANOVA) are presented in Table II. Participants achieved the maximal swimming power at $4.45 \pm 0.86 \mathrm{~kg}$ and it corresponded to $31.18 \pm 7.98 \%$ of the maximal load.

The RFD showed to be higher after DLWU $(34.52 \pm 16.55)$ compared to SWU $(31.29 \pm 13.70 ; \mathrm{P}=0.032)$, but no differences were observed between SWU and INCTEST. The force, Accel, Imp $\mathrm{IEL}_{\mathrm{RE}}$ and power values were lower in DLWU compared to SWU (Table I). No differences were observed on those variables when compared SWU with INCTEST, except for power, which showed to be higher in INCTEST $(51.38 \pm 14.93 \mathrm{~W})$ than obtained in SWU $(49.98 \pm 15.40 \mathrm{~W})(\mathrm{P}=0.037)$.

The semi-tethered swimming velocity, stroke-length,

TABLE I.-Mean, SD, 95\% confident intervals, relative changes (\% $\%$ ), P value, and Effect Sizes of the kinetic and kinematic variables collected in semi-tethered resisted swimming $(S T R S)(N .=20)$.

\begin{tabular}{|c|c|c|c|c|c|c|c|c|c|}
\hline \multirow{2}{*}{ Variable } & \multirow{2}{*}{ SWU } & \multirow{2}{*}{ DLWU } & \multirow{2}{*}{ INCTEST } & \multicolumn{3}{|c|}{ SWU vs. DLWU } & \multicolumn{3}{|c|}{ SWU vs. INCTEST } \\
\hline & & & & $\% \Delta$ & P value & Effect size & $\% \Delta$ & P value & Effect size \\
\hline Force (N.) & $\begin{array}{c}42.95 \pm 10.15 \\
(35.63-50.16)\end{array}$ & $\begin{array}{c}41.82 \pm 9.87^{*} \\
(34.86-48.99)\end{array}$ & $\begin{array}{c}43.22 \pm 10.13 \\
(35.97-50.47)\end{array}$ & $-2.60 \%$ & 0.001 & -0.11 & $0.63 \%$ & 0.052 & 0.02 \\
\hline $\operatorname{Accel}\left(\mathrm{m} / \mathrm{s}^{2}\right)$ & $\begin{array}{c}0.23 \pm 0.08 \\
(0.17-0.29)\end{array}$ & $\begin{array}{l}0.16 \pm 0.05^{*} \\
(0.13-0.20)\end{array}$ & $\begin{array}{c}0.25 \pm 0.08 \\
(0.19-0.31)\end{array}$ & $-30.41 \%$ & 0.049 & -1.04 & $6.88 \%$ & 0.117 & 0.25 \\
\hline $\operatorname{Imp}_{\mathrm{REL}}(\mathrm{N} \cdot \mathrm{s})$ & $\begin{array}{c}4.41 \pm 1.54 \\
(3.31-5.51)\end{array}$ & $\begin{array}{l}3.49 \pm 1.39 * \\
(2.50-4.49)\end{array}$ & $\begin{array}{c}4.48 \pm 1.58 \\
(3.35-5.62)\end{array}$ & $-20.84 \%$ & 0.023 & -0.62 & $1.56 \%$ & 0.078 & 0.04 \\
\hline Power (w) & $\begin{array}{c}49.98 \pm 15.40 \\
(38.96-61.00)\end{array}$ & $\begin{array}{l}42.48 \pm 12.95^{*} \\
(33.21-51.75)\end{array}$ & $\begin{array}{l}51.38 \pm 14.93 * \\
(40.70-62.06)\end{array}$ & $-15.01 \%$ & 0.002 & -0.87 & $2.72 \%$ & 0.037 & 0.12 \\
\hline RFD (N./s) & $\begin{array}{c}31.29 \pm 13.70 \\
(21.49-41.09)\end{array}$ & $\begin{array}{l}34.52 \pm 16.55^{*} \\
(21.97-47.08)\end{array}$ & $\begin{array}{c}31.79 \pm 13.49 \\
(22.14-41.44)\end{array}$ & $9.35 \%$ & 0.032 & 0.21 & $1.59 \%$ & 0.241 & 0.03 \\
\hline Velocity (m/s) & $\begin{array}{c}1.17 \pm 0.12 \\
(1.08-1.26)\end{array}$ & $\begin{array}{l}1.01 \pm 0.15^{*} \\
(0.90-1.11)\end{array}$ & $\begin{array}{l}1.21 \pm 0.14^{*} \\
(1.11-1.32)\end{array}$ & $-13.67 \%$ & 0.001 & -1.17 & $3.06 \%$ & 0.041 & 0.30 \\
\hline $\mathrm{SR}$ (cyc/min) & $\begin{array}{c}61.56 \pm 7.07 \\
(56.64-66.87)\end{array}$ & $\begin{array}{c}64.70 \pm 9.84 * \\
(57.66-71.74)\end{array}$ & $\begin{array}{c}61.43 \pm 7.27 \\
(56.23-66.64)\end{array}$ & $5.10 \%$ & 0.044 & 0.31 & $-1.14 \%$ & 0.204 & -0.01 \\
\hline $\mathrm{SL}(\mathrm{m})$ & $\begin{array}{c}1.21 \pm 0.15 \\
(1.11-1.33)\end{array}$ & $\begin{array}{l}0.97 \pm 0.20^{*} \\
(0.83-1.12)\end{array}$ & $\begin{array}{c}1.23 \pm 0.16 \\
(1.11-1.35)\end{array}$ & $-19.83 \%$ & $<0.001$ & -1.35 & $2.34 \%$ & 0.184 & 0.12 \\
\hline $\mathrm{DC}(\mathrm{m})$ & $\begin{array}{c}5.77 \pm 0.72 \\
(5.25-6.29)\end{array}$ & $\begin{array}{l}4.73 \pm 1.09^{*} \\
(3.94-5.51)\end{array}$ & $\begin{array}{c}5.95 \pm 0.88 \\
(5.31-6.59)\end{array}$ & $-18.02 \%$ & $<0.001$ & -1.12 & $3.13 \%$ & 0.059 & 0.22 \\
\hline $\mathrm{T} 5 \mathrm{~m}(\mathrm{~s})$ & $\begin{array}{c}4.23 \pm 0.57 \\
(3.82-4.64)\end{array}$ & $\begin{array}{l}5.22 \pm 0.88^{*} \\
(4.59-5.86)\end{array}$ & $\begin{array}{c}4.19 \pm 0.56 \\
(3.79-4.60)\end{array}$ & $23.40 \%$ & 0.003 & 1.33 & $-0.76 \%$ & 0.087 & -0.07 \\
\hline IVV (\%) & $\begin{array}{c}45.98 \pm 9.63 \\
(37.36-54.84)\end{array}$ & $\begin{array}{c}37.95 \pm 7.91 \\
(30.61-43.94)\end{array}$ & $\begin{array}{c}44.81 \pm 8.31 \\
(36.99-52.42)\end{array}$ & $-17.46 \%$ & $<0.001$ & -0.91 & $-2.54 \%$ & 0.158 & -0.13 \\
\hline
\end{tabular}

SWU: Standard warm-up; DLWU: dry-land warm-up; INCTEST: incremental test.

*Significant differences $(\mathrm{P}<0.05)$. 


\section{COPYRIGHT ${ }^{\circledR} 2020$ EDIZIONI MINERVA MEDICA}

PULL-OVER POTENTIATION IN SEMI-TETHERED SWIMMING

CUENCA-FERNÁNDEZ

TABLE II.-Pearson's correlation coefficients of the kinetic and kinematic variables collected in semi-tethered resisted swimming $(N .=20)$.

\begin{tabular}{|c|c|c|c|c|c|c|c|c|c|}
\hline \multirow{2}{*}{ Variable } & & \multicolumn{2}{|c|}{ Stroke rate } & \multicolumn{2}{|c|}{ Stroke length } & \multicolumn{2}{|c|}{ Distance covered } & \multicolumn{2}{|c|}{ Time 5M } \\
\hline & & $\mathrm{r}$ & $\mathrm{P}$ & $\mathrm{r}$ & $\mathrm{P}$ & $\mathrm{r}$ & $\mathrm{P}$ & $\mathrm{r}$ & $\mathrm{P}$ \\
\hline \multirow{7}{*}{ SWU } & Velocity & - & - & 0.821 & 0.004 & 0.710 & 0.020 & -0.987 & 0.001 \\
\hline & Force & 0.652 & 0.038 & - & - & - & - & -0.610 & 0.046 \\
\hline & Accel & - & - & - & - & - & - & - & - \\
\hline & $\operatorname{Imp}_{\text {REL }}$ & - & - & 0.651 & 0.039 & - & - & -0.709 & 0.027 \\
\hline & Power & - & - & 0.607 & 0.049 & - & - & -0.798 & 0.008 \\
\hline & RFD & - & - & - & - & - & - & - & - \\
\hline & IVV & 0.615 & 0.050 & -0.697 & 0.025 & -0.606 & 0.042 & - & - \\
\hline \multirow[t]{7}{*}{ DLWU } & Velocity & - & - & 0.743 & 0.014 & 0.742 & 0.014 & -0.905 & 0.000 \\
\hline & Force & - & - & - & - & - & - & - & - \\
\hline & Accel & - & - & - & - & - & - & -0.637 & 0.047 \\
\hline & $\operatorname{Imp}_{\text {REL }}$ & - & - & 0.762 & 0.027 & - & - & - & - \\
\hline & Power & - & - & - & - & - & - & -0.676 & 0.032 \\
\hline & RFD & - & - & - & - & - & - & - & - \\
\hline & IVV & - & - & - & - & - & - & - & - \\
\hline \multirow[t]{7}{*}{ INCTEST } & Velocity & - & - & 0.836 & 0.003 & 0.711 & 0.021 & -0.989 & 0.000 \\
\hline & Force & 0.673 & 0.033 & - & - & - & - & -0.644 & 0.044 \\
\hline & Accel & - & - & - & - & - & - & - & - \\
\hline & $\operatorname{Imp}_{\mathrm{REL}}$ & - & - & 0.664 & 0.036 & - & - & -0.729 & 0.017 \\
\hline & Power & - & - & 0.618 & 0.047 & - & - & -0.810 & 0.005 \\
\hline & RFD & - & - & - & - & - & - & - & - \\
\hline & IVV & 0.631 & 0.048 & -0.707 & 0.022 & -0.656 & 0.039 & - & - \\
\hline
\end{tabular}

SWU: Standard warm-up; DLWU: dry-land warm-up; INCTEST: incremental test.

distance covered and IVV were lower in DLWU compared to SWU (Table I), but the statistical analyses not showed differences between SWU and INCTEST. The values of stroke-rate $(64.70 \pm 9.84 \mathrm{~Hz})$ and the time to cover $5 \mathrm{~m}(5.22 \pm 0.88 \mathrm{~s})$, were higher in DLWU in comparison to SWU (SR: $61.56 \pm 7.07 \mathrm{~Hz} ; \mathrm{P}=0.044)(4.23 \pm 0.57 \mathrm{~s}$; $\mathrm{P}=0.003)$. No differences were obtained in those variables between SWU and INCTEST.

At least 3 kinetic variables obtained correlations with the kinematic variables of the STRS efforts performed. On one hand, velocity obtained strong correlations with SR, SL and T5m in SWU, DLWU and INCTEST (Table II). On the other hand, Imp $\mathrm{R}_{\mathrm{REL}}$ correlated with SL in the 3 protocols, with the highest value obtained after DLWU $(r=0.76)$. At last, Power correlated with T5m ( $\mathrm{r}=\sim-0.67--0.81)$ with higher values obtained in INCTEST. Other variables such as force obtained moderate correlations with SR and T5m in INCTEST and SWU, while IVV correlated with SR, SL and DC in the same aforementioned protocols. Finally, Accel correlated with T5m ( $\mathrm{r}=-0.63)$ only in DLWU (Table II).

\section{Discussion}

The aim of this study was to investigate whether muscular performance could be improved after a dry-land or an aquatic warm-up in semi-tethered resisted swimming
(STRS). It was hypothesized that testing swimmer kinetics variables through STRS could provide a real picture of the biological changes generated in the muscular capacity after a resistance warm-up to discern between the presence or not of performance enhancements (PAPE). The results showed an increase in RFD and stroke-rate after high-resistance pull-over repetitions; however, other variables such as velocity and distance covered showed deterioration in performance. Therefore, potentiation responses were present after the resistance warm-up, but they were not accompanied by PAPE effects.

The simple adaptation of a linear-encoder system designed to measure performance in dry-land conditions, allowed us to also measure performance in the water. ${ }^{12}$ Considering that semi-tethered swimming allows displacement and movement velocity has been shown to be a predictor of loading intensity and strength capability in resistance training, ${ }^{36}$ our STRS protocol showed to be sensitive in obtaining valuable information about the neuromuscular changes produced on swimmers to understand how kinetic changes may affect kinematic measurements. For that reason, and while there is no possibility of acquiring another more recommended equipment, 16,28 this system could be an equally valid alternative to test swimmers despite the potential risk of altering the swimming patterns. ${ }^{12,}{ }^{30}$ In fact, considering that in-water re- 


\section{COPYRIGHT $^{\odot} 2020$ EDIZIONI MINERVA MEDICA}

sisted procedures are usually integrated into swimming programs, ${ }^{10,27}$ the participants would be familiar with this testing procedure.

An increase in RFD was obtained after the dry-land warm-up (Table I). However, it was not accompanied by performance enhancements. The RFD is the ability to increase force or torque as quickly as possible during a rapid voluntary contraction conducted from a low or resting level. ${ }^{37}$ It has been reported to increase after resistance training:33 to be sensitive to acute changes in neuromuscular function, ${ }^{38}$ and to be potentially governed by different physiological mechanisms such as PAP. ${ }^{23}$ However, there are several reasons to discuss why it would be inappropriate to link the effects provided by DLWU with this responsemechanism. First of all, as muscle biopsy was not conducted to verify the phosphorylation levels, ${ }^{23}$ it prevented a conclusion favoring the presence of PAP-effects. Thus, our results were based on an alternative interpretation provided by the encoder dynamic recordings (Figure 2).

Apparently, the peaks reached in force and velocity after SWU were not achieved after DLWU (Figure 2), which led to lower average values on these variables (Table I). At this point, it is important to note that the RFD was calculated as the slope of the force-time curve $(\Delta$ Force/ $\Delta$ Time). ${ }^{33}$ Therefore, the reduction of the slope within the stroke-cycle produced a shorter time to reach the peak, which could result in higher RFD. This fact has been reported in other studies, where apparent RFD increases did not yield performance enhancements. ${ }^{5,37}$ In fact, considering that the differences within the stroke-cycle are dependent on the ability to overcome the resistance to generate efficient propulsion, 34,39 the significant reduction obtained in IVV after DLWU could indicate that a lower energy was transferred into the water as a consequence of the warm-up and this produced a reduction of acceleration with an apparent increase in RFD.

The nature of a swimmer's locomotion lies in the hydrodynamic reaction forces created by the swimmer's limb movements to overcome water resistance. ${ }^{17}$ Attending to the correlations found between velocity and stroke-length in the STRS efforts (Table II), the swimmers achieving a larger arm-stroke were the ones obtaining higher velocity $(r=0.73-0.84)$. In fact, the low values of $\operatorname{Imp}_{\text {REL }}$ obtained in DLWU combined with the reduction of the distance covered and the increase of the Stroke-rate seemed to support that fact, indicating that every arm-stroke was shortened and less efficient (Table I).12, 30 Therefore, although an increase on the arm-pull speed could be a consequence of neuromuscular potentiation adaptations, it could reduce the lateral and/or sculling like movements in the arm-stroke trajectory, producing a slippery effect on the stroke cycle. ${ }^{9}$

This conclusion agrees with the one already proposed by Barbosa et al. ${ }^{16}$ The increases obtained on the arm-pull thrust reflected an acute enhancement of the neuromuscular mechanism. However, the arm-pull thrust does not essentially represent the effective propulsive force generated by the body, but the increase of the force conveyed per stroke against the water. Thus, although potentiation responses were obtained and it also produced a moderate increase in swimming velocity $(\sim 2 \%)$, it should be noted that little or no benefit could be obtained by increasing upper limbs performance in swimming if this is not in line with an increase of the effective propulsive impulse.

In any case, potentiation responses are always accompanied by varying fatigue manifestations. ${ }^{2}, 40$ Studies have shown that a reduced capacity to generate propulsive impulse per stroke (i.e. fatigue), decreases stroke-length and increases stroke-rate, ${ }^{30,41}$ similar to that obtained after DLWU. Moreover, as the correlation analysis demonstrated that swimmers producing higher Velocity, $\operatorname{Imp}_{\mathrm{REL}}$ and power achieved also higher stroke-length, then such relationship between kinetics and kinematics was confirmed. Therefore, although it was nevertheless demonstrated that an uncontrolled improvement in muscular capacity could also produce a detrimental biomechanical consequence in swimming performance, it is not disregarded that the lack of PAPE effects may be a consequence of fatigue prevalence as reported in previous studies. ${ }^{13,14,27}$

On the other hand, it has been debated whether the effects in power or strength provided by dry-land CEs could be transferred to the water. ${ }^{15}$ In swimming, the hand surface area and upper-body strength have shown to predict upper limb and full stroke cycle thrust, respectively. ${ }^{29}$ Concretely, the upper-body strength has been assessed in different ways both in pulling as in pushing exercises since these exercises recruits several muscles related to front crawl stroke. ${ }^{9-12,}{ }^{29}$ However, the optimal intensity of the CEs is still uncertain. Some authors, ${ }^{6,}{ }^{42}$ rationalized that a potentiation strategy should be maximal or near maximal intensity to increase motor unit activation ( $\geq 85-90 \%$ $1 \mathrm{RM}$ ), based on the fact that the speed of force transmission through a material is influenced by the material stiffness. ${ }^{37}$ On this line, specific low-volume, high-force resistance training has been proposed as an optimal approach to transfer strength gains to swimming performance. 15,20

However, a very heavy resistance set may temporarily result in some increased stiffness of the musculo-tendi- 


\title{
COPYRIGHT $^{(\odot)} 2020$ EDIZIONI MINERVA MEDICA
}

\author{
PULL-OVER POTENTIATION IN SEMI-TETHERED SWIMMING
}

CUENCA-FERNÁNDEZ

nous unit and specifically the series elastic component (SEC) than what would be optimal considering the lighter resistance to be overcome. ${ }^{43}$ According to Baker, ${ }^{8}$ the use of very heavy resistances in contrast loading for the upper body may not be as effective as for the lower body, possibly due to the smaller muscle mass involved. Moreover, since the velocities reached on the CE were clearly slow, the required innervated fibers may not be appropriate, creating an erroneous stimulation pattern.8,24 This motor pattern interference has been identified in previous literature as "perseveration," since the motor gross pattern of a task (e.g. cycling) perseveres, while a subsequent task of a different modality (e.g. running) is commenced. 44,45 Therefore, at this point it is worth mentioning that the dry-land CE was not included in either SWU or INCTEST.

The maximal swimming power during INCTEST was achieved at $4.45 \pm 0.86 \mathrm{~kg}$, which means that at least 3 to 5 STRS efforts were performed by every participant before establishing such category. However, the results did not seem to be affected by fatigue but by PAPE effects. ${ }^{26}$ According to some authors, temperature changes could explain velocity and power-dependent effects in muscle mechanisms. ${ }^{4,46,47}$ Therefore, it is not surprising that the high volume of work of INCTEST produced a reasonable increase in muscle temperature. However, such argument could make questionable the deterioration encountered in DWLU, given that this protocol also received a considerable amount of work (i.e. SWU followed by DLWU). Possibly, some tension sensitive receptors such as the Golgi tendon organ could account for a consequent change in power output reducing their negative inhibitory feedback after a moderate increase in resistance. ${ }^{8}$ These neurological adaptations may have temporarily resulted in a favorable increase in SEC stiffness in the ensuing STRS efforts that may have triggered PAPE responses. $48-50$

At last, another possible explanation may reside in the rest time given between efforts. ${ }^{12,13,26}$ Although the deviating time course of performance enhancement is an individually regulated response that depends on the training experience and the nature of the participants muscle fiber composition, 24,40 an inappropriate time of rest would diminish or mask the effects of PAPE. 13, 27 Nevertheless, some mechanisms such as the vascular bed dilation/blood muscle perfusion and the increased muscle temperature are only possible after several minutes (4 to $10 \mathrm{~min}) .4,5,47$ Therefore, the rest period chosen (6 min), was presumably adequate to allow performance enhance- ments in INCTEST. In such case, the effort displaying the maximal power gathered performance enhancements prompted by the efforts that preceded it (i.e. at $10-30 \%$ of the maximal load).

\section{Limitations of the study}

This study presented some limitations, as the STRS encoder recordings may not just be from the arm action throughout the underwater stroke, but also from the leg action. In any case, according to a previous research, ${ }^{15}$ the majority of propulsive forces in swimming are produced from the upper body, with strong correlations between upper body strength and sprint performance. Future research should provide more information about this issue and continue to attempt CEs (both in aquatic and in dry land conditions) to transfer the effects of PAPE to cyclic sports such as swimming. The inclusion of differentiated arm movements, rotation of the body, or low loaded conditioning exercises may allow faster movements and, therefore, better adaptations.

\section{Conclusions}

This study showed RFD potentiation responses on swimmers after a dry-land resistance warm-up. However, they did not improve swimming performance, possibly due to alterations in the biomechanics of the stroke. In any case, it seems that swimmers may benefit more from submaximal prolonged conditioning activities conducted in the water to develop high power and propulsive impulse, due to adaptive changes of the neuromuscular system. Specifically, attaining a high propulsive impulse could have a positive influence in other kinematic variable such as stroke-length, which is important to achieve a good race result.

\section{References}

1. Cuenca-Fernández F, Smith IC, Jordan MJ, MacIntosh BR, LópezContreras G, Arellano R, et al. Nonlocalized postactivation performance enhancement (PAPE) effects in trained athletes: a pilot study. Appl Physiol Nutr Metab 2017;42:1122-5.

2. Rassier DE, Macintosh BR. Coexistence of potentiation and fatigue in skeletal muscle. Braz J Med Biol Res 2000;33:499-508.

3. Wang L, Bahadir A, Kawai M. High ionic strength depresses muscle contractility by decreasing both force per cross-bridge and the number of strongly attached cross-bridges. J Muscle Res Cell Motil 2015;36:227-41.

4. González-Alonso J, Quistorff B, Krustrup P, Bangsbo J, Saltin B. Heat production in human skeletal muscle at the onset of intense dynamic exercise. J Physiol 2000;524:603-15.

5. Blazevich AJ, Babault N. Post-activation Potentiation Versus Post-activation Performance Enhancement in Humans: Historical Perspective, Underlying Mechanisms, and Current Issues. Front Physiol 2019;10:1359. 


\section{COPYRIGHT $^{\odot} 2020$ EDIZIONI MINERVA MEDICA}

6. Güllich A, Schmidtbleicher D. MVC-induced short-term potentiation of explosive force. New studies in athletics 1996;11:67-84.

7. Botcazou M, Gratas-Delamarche A, Allain S, Jacob C, Bentué-Ferrer $\mathrm{D}$, Delamarche $\mathrm{P}$, et al. Influence de la phase du cycle menstruel sur les réponses en catécholamines à l'exercice de sprint chez la femme. Appl Physiol Nutr Metab 2006;31:604-11. English.

8. Baker D. Acute effect of alternating heavy and light resistances on power output during upper-body complex power training. J Strength Cond Res 2003;17:493-7.

9. Dominguez-Castells R, Izquierdo M, Arellano R. An updated protocol to assess arm swimming power in front crawl. Int J Sports Med 2013;34:324-9.

10. Pérez-Olea JI, Valenzuela PL, Aponte C, Izquierdo M. Relationship Between Dryland Strength and Swimming Performance: Pull-Up Mechanics as a Predictor of Swimming Speed. J Strength Cond Res 2018;32:1637-42.

11. Morouço P, Neiva H, González-Badillo JJ, Garrido N, Marinho DA, Marques MC. Associations between dry land strength and power measurements with swimming performance in elite athletes: a pilot study. J Hum Kinet 2011;29A:105-12.

12. Cuenca-Fernández F, Gay A, Ruiz-Navarro JJ, Arellano R. The effect of different loads on semi-tethered swimming and its relationship with dry-land performance variables. Int J Perform Anal Sport 2020;1-17.

13. Cuenca-Fernández F, Ruiz-Teba A, López-Contreras G, Arellano R. Effects of 2 Types of Activation Protocols Based on Postactivation Potentiation on 50-m Freestyle Performance. J Strength Cond Res 2018. [Epub ahead of print]

14. Sarramian VG, Turner AN, Greenhalgh AK. Effect of postactivation potentiation on fifty-meter freestyle in national swimmers. J Strength Cond Res 2015;29:1003-9.

15. Crowley E, Harrison AJ, Lyons M. The impact of resistance training on swimming performance: A systematic review. Sports Med 2017; 47:2285-307.

16. Barbosa TM, Yam JW, Lum D, Balasekaran G, Marinho DA. Armpull thrust in human swimming and the effect of post-activation potentiation. Sci Rep 2020;10:8464.

17. Vorontsov A, Rumyantsev V. Propulsive forces in swimming In Zatsiorsky VM, editor. Biomechanics in Sport: Performance Enhancement and Injury Prevention. Oxford: Blackwell Science; 2000. p.205-31.

18. Andersen JT, Sinclair PJ, McCabe CB, Sanders RH. Kinematic Differences in Shoulder Roll and Hip Roll at Different Front Crawl Speeds in National Level Swimmers. J Strength Cond Res 2020;34:20-5.

19. Loebbecke A, Mittal R. Comparative analysis of thrust production for distinct arm-pull styles in competitive swimming. J Biomech Eng $2012 ; 134$.

20. Morais JE, Silva AJ, Garrido ND, Marinho DA, Barbosa TM. The transfer of strength and power into the stroke biomechanics of young swimmers over a 34-week period. Eur J Sport Sci 2018;18:787-95.

21. Amaro N, Marinho DA, Batalha N, Marques MC, Morouço P. Reliability of tethered swimming evaluation in age group swimmers. J Hum Kinet 2014;41:155-62.

22. Prieske O, Behrens M, Chaabene H, Granacher U, Maffiuletti NA Time to Differentiate Postactivation "Potentiation" from "Performance Enhancement" in the Strength and Conditioning Community. Sports Medicine; 2020 [Internet]. Available from: https://link.springer.com/article/10.1007/s40279-020-01300-0 [cited 2020, Jul 8].

23. Vandenboom R. Modulation of skeletal muscle contraction by myosin phosphorylation. Compr Physiol 2016;7:171-212.

24. Tillin NA, Bishop D. Factors modulating post-activation potentiation and its effect on performance of subsequent explosive activities. Sports Med 2009;39:147-66.

25. Dalamitros AA, Mavridis G, Semaltianou E, Loupos D, Manou V. Psychophysiological and performance-related responses of a potentia- tion activity in swimmers of different competitive levels. Physiol Behav 2019;204:106-11.

26. Hancock AP, Sparks KE, Kullman EL. Postactivation potentiation enhances swim performance in collegiate swimmers. J Strength Cond Res 2015;29:912-7.

27. Barbosa AC, Barroso R, Andries O Jr. Post-activation Potentiation in Propulsive Force after Specific Swimming Strength Training. Int J Sports Med 2016;37:313-7.

28. Ng F, Wen Yam J, Lum D, Barbosa TM. Human thrust in aquatic environment: the effect of post-activation potentiation on flutter kick. J Adv Res 2019;21:65-70.

29. Morais JE, Marques MC, Rodríguez-Rosell D, Barbosa TM, Marinho DA. Relationship between thrust, anthropometrics, and dry-land strength in a national junior swimming team. Phys Sportsmed 2019;1-8.

30. Dominguez-Castells R, Arellano R. Effect of different loads on stroke and coordination parameters during freestyle semi-tethered swimming. $\mathrm{J}$ Hum Kinet 2012;32:33-41.

31. Ferguson B. ACSM's guidelines for exercise testing and prescription 9th Ed. 2014. J Can Chiropr Assoc 2014;58:328.

32. Shionoya A, Shibukura T, Koizumi M, Shimizu T, Tachikawa K, Hasegawa M, et al. Development of ergometer attachment for power and maximum anaerobic power measurement in swimming. Appl Human Sci 1999; 18:13-21

33. Aagaard P, Simonsen EB, Andersen JL, Magnusson P, DyhrePoulsen P. Increased rate of force development and neural drive of human skeletal muscle following resistance training. J Appl Physiol (1985) 2002;93:1318-26.

34. Morouço PG, Barbosa TM, Arellano R, Vilas-Boas JP. Intracyclic Variation of Force and Swimming Performance. Int J Sports Physiol Perform 2018;13:897-902.

35. Cohen J. Statistical Power Analysis for the Behavioral Sciences. Second edition. Hillsdale, NJ: Lawrence Erlbaum Associates; 1988.

36. González-Badillo JJ, Sánchez-Medina L. Movement velocity as a measure of loading intensity in resistance training. Int J Sports Med 2010;31:347-52.

37. Maffiuletti NA, Aagaard P, Blazevich AJ, Folland J, Tillin N, Duchateau J. Rate of force development: physiological and methodological considerations. Eur J Appl Physiol 2016;116:1091-116.

38. Peñailillo L, Blazevich A, Numazawa $H$, Nosaka $K$. Rate of force development as a measure of muscle damage. Scand J Med Sci Sports $2015 ; 25: 417-27$

39. Barbosa TM, Morouço PG, Jesus S, Feitosa WG, Costa MJ, Marinho $\mathrm{DA}$, et al. The interaction between intra-cyclic variation of the velocity and mean swimming velocity in young competitive swimmers. Int J Sports Med 2013;34:123-30.

40. Seitz LB, Haff GG. Factors Modulating Post-Activation Potentiation of Jump, Sprint, Throw, and Upper-Body Ballistic Performances: A Systematic Review with Meta-Analysis. Sports Med 2016;46:231-40

41. Alberty M, Sidney M, Pelayo P, Toussaint HM. Stroking characteristics during time to exhaustion tests. Med Sci Sports Exerc 2009;41:637-44

42. Young WB, Jenner A, Griffiths K. Acute enhancement of power performance from heavy load squats. J Strength Cond Res 1998;12:82-4.

43. Wilson GJ, Murphy AJ, Giorgi A. Weight and plyometric training: effects on eccentric and concentric force production. Can J Appl Physiol 1996;21:301-15.

44. Kay AD, Husbands-Beasley J, Blazevich AJ. Effects of contract-relax, static stretching, and isometric contractions on muscle-tendon mechanics. Med Sci Sports Exerc 2015;47:2181-90.

45. Gottschall JS, Palmer BM. The acute effects of prior cycling cadence on running performance and kinematics. Med Sci Sports Exerc 2002;34:1518-22. 


\section{COPYRIGHT $^{\circledR} 2020$ EDIZIONI MINERVA MEDICA}

46. Sargeant AJ. Effect of muscle temperature on leg extension force and short-term power output in humans. Eur J Appl Physiol Occup Physiol 1987;56:693-8.

47. Nuzzo JL, Barry BK, Gandevia SC, Taylor JL. Acute Strength Training Increases Responses to Stimulation of Corticospinal Axons. Med Sci Sports Exerc 2016;48:139-50.

48. Zhang D, Chippada U, Jordan K. Effect of the structural water on the mechanical properties of collagen-like microfibrils: a molecular dynamics Study. Ann Biomed Eng 2007;35:1216-30.

49. Girold S, Calmels P, Maurin D, Milhau N, Chatard JC. Assisted and resisted sprint training in swimming. J Strength Cond Res 2006;20:547-54.

50. Delecluse C, Van Coppenolle H, Willems E, Van Leemputte M, Diels $\mathrm{R}$, Goris $\mathrm{M}$. Influence of high-resistance and high-velocity training on sprint performance. Med Sci Sports Exerc 1995;27:1203-9.

Conflicts of interest.-The authors certify that there is no conflict of interest with any financial organization regarding the material discussed in the manuscript. Funding.- This study was supported by grants awarded by the Ministry of Economy, Industry and Competitiviness (Spanish Agency of Research) and the European Regional Development Fund (ERDF); DEP2014-59707-P "SWIM: Specific Water Innovative Measurements applied to the development of International Swimmers in Short Swimming Events (50 and $100 \mathrm{~m}$ ), by the Ministry of Science, Innovation and Universities (Spanish Agency of Research) and the European Regional Development Fund (ERDF); PGC2018-102116-B-I00 "SWIM II: Specific Water Innovative Measurements: Applied to the performance improvement" and the Spanish Ministry of Education, Culture and Sport: FPU17/02761 grant. This article is a part of an international thesis belonging to the Program of PhD in Biomedicine (B11.56.1), from the University of Granada, Granada (Spain).

Authors' contributions.-All authors read and approved the final version of the manuscript.

Acknowledgements.-Authors acknowledge all the swimmers who voluntarily participated in this research.

History. -Article first published online: June 30, 2020. - Manuscript accepted: June 22, 2020. - Manuscript revised: June 12, 2020. - Manuscript received: April 29, 2020. 